\title{
Bio-Inspired Water Strider Robots with Microfabricated Functional Surfaces
}

\author{
Kenji Suzuki \\ Kogakuin University \\ Japan
}

\section{Introduction}

In recent years, there has been considerable interest in insect-inspired miniature robots. Through evolutionary processes, insects have prospered by adapting themselves to diverse environments. The number of species of insects is approximately one million, which comprises approximately two-thirds of all species of animals. By taking advantage of scaling effects, insects have acquired unique locomotive abilities, such as hexapedal walking, climbing on walls, jumping, and flying by flapping, that markedly extend their fields of activity. The working principles behind these behaviours are considered to be highly efficient and optimized for miniature systems. Therefore, they provide alternative design rules for developing smart and advanced microrobotic mechanisms. For example, the flapping motion of insect wings has been investigated for micromechanical flying robots (Suzuki, et al., 1994; Wood, 2008). This chapter focuses on the locomotion of water striders. This motion is dependent on surface tension. Recent studies have demonstrated the mechanisms that enable insects to stay, as well as move, on water. Furthermore, various kinds of miniature robots that are able to move on water have been developed. Hu et al. identified the mechanism of the momentum transfer that is responsible for water strider locomotion and proposed a mechanical water strider driven by elastic thread ( $\mathrm{Hu}$, et al., 2003). Gao et al. showed that the legs of water striders are covered with thousands of tiny hairs that have fine nanoscale grooves (Gao \& Jiang, 2004). These hierarchical micro- and nanostructures create super-hydrophobic surfaces. Suhr et al. developed a water strider robot that is driven in one of its resonant modes by using unimorph piezoelectric actuators (Suhr, et al., 2005). Song et al. numerically calculated the statics of rigid and flexible supporting legs (Song, et al., 2006; Song, et al., 2007a) and developed a non-tethered water strider robot using two miniature DC motors and a lithium-polymer battery (Song \& Sitti, 2007b). The locomotion mechanisms of fisher spider (Suter \& Wildman, 1997; Suter, et al., 1999) and basilisk lizard (Glasheen \& McMahon, 1996a; 1996b) on the surface of water were studied. A robot that mimics the water running ability of the basilisk lizard was also developed (Floyd, et al., 2006; Floyd \& Sitti, 2008).

The present authors (Suzuki, et al., 2007) have fabricated hydrophobic supporting legs with microstructured surfaces utilizing MEMS (microelectromechanical systems) techniques, and 


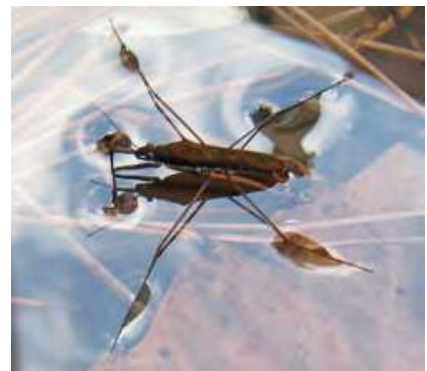

(a) Water strider

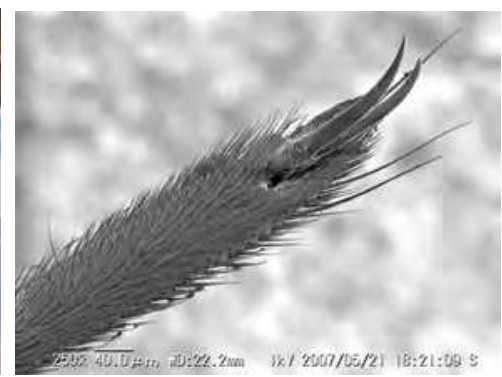

(b) Tip of its leg

Fig. 1.The water strider, used as the robot model

developed non-tethered water strider robots with MEMS-structured legs. In this study, equations for the forces acting on a partially submerged supporting leg were derived analytically, and the effects of the diameter and contact angle of the leg on the forces were investigated. Then, various kinds of hydrophobic supporting legs with and without microfabricated surfaces were prepared, and the lift and pull-off forces on the water surface were measured to verify the theoretical analyses. In addition, two non-tethered mechanisms for water strider robots with microfabricated legs were created to demonstrate autonomous locomotion on the surface of water.

\section{Theoretical model of a supporting leg}

\subsection{Lift force}

Water striders can stay and move on the surface of water by primarily using surface tension force. Figure 1 shows a water strider on a water surface and an SEM image of the tip of its leg. The leg is covered with tiny hairs, which improve the hydrophobicity and reduce the drag force. In this section, equations of the buoyancy and surface tension forces acting on a partially submerged cylindrical leg are analytically derived

Figure 2 shows a two-dimensional model of the supporting leg. We assume that the leg is a long, rigid cylinder of uniform material with radius $r$ and contact angle $\theta_{c}$. The vertical lift force $F$ acting on the leg of unit length consists of a buoyancy force $F_{B}$ and a force due to surface tension $F_{S}$.

$$
F=F_{B}+F_{S}
$$
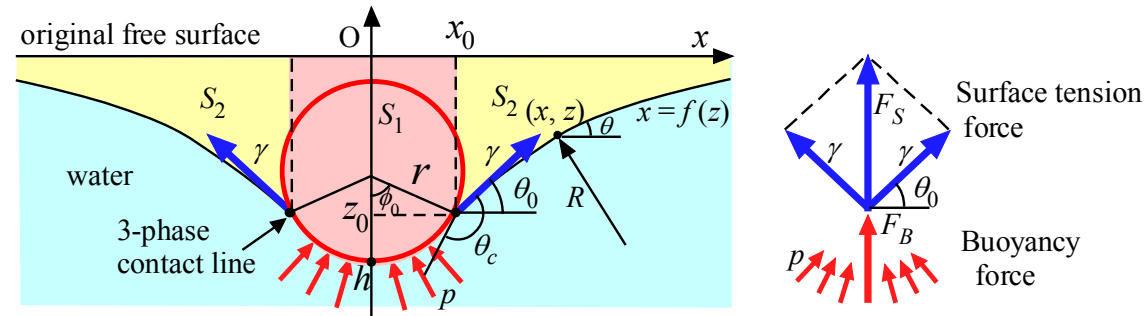

Fig. 2. Two dimensional model of the supporting leg. 
The buoyancy force $F_{B}$ is deduced by integrating the vertical component of hydrostatic pressure $p$ over the body area in contact with the water. The force due to surface tension $F_{S}$ is the vertical component of the surface tension per unit length $\gamma$ acting on the three-phase contact line. Keller demonstrated that $F_{B}$ and $F_{S}$ are equal to the weights of water displaced inside and outside of the three-phase contact line, respectively (Keller, 1998). That is, $F_{B}$ is proportional to the area $S_{1}$, shown in Fig. 2, and $F_{S}$ is proportional to the area $S_{2}$.

$$
\begin{gathered}
F_{B}=\int_{0}^{\phi_{0}} p \cos \phi \cdot r d \phi=\rho g S_{1} \\
=\rho g\left(-2 z_{0} r \sin \phi_{0}-r^{2} \sin \phi_{0} \cos \phi_{0}+r^{2} \phi_{0}\right) \\
F_{S}=2 \gamma \sin \theta_{0}=\rho g S_{2}
\end{gathered}
$$

where $\phi_{0}$ is the submerged angle and $\theta_{0}$ is the slope of the water surface, as shown in Fig. 2. The subscript " 0 " represents the value on the three-phase contact line. The relationship between $\phi_{0}$ and $\theta_{0}$ is given by:

$$
\phi_{0}=\pi+\theta_{0}-\theta_{c}
$$

From the Young-Laplace equation, hydrostatic pressure on the surface of water is:

$$
p=-\rho g z=\frac{\gamma}{R}
$$

where $R$ is the radius of curvature of the water surface. The governing equation of the water's surface profile as a function of $z, x=f(z)$ is given by

$$
\frac{\rho g z}{\gamma}=\operatorname{Sign}(z) \frac{f^{\prime \prime}(z)}{\left(1+f^{\prime}(z)^{2}\right)^{\frac{3}{2}}}
$$

The boundary conditions for $f(z)$ are

$$
f(0)=\infty, f\left(z_{0}\right)=x_{0}=r \sin \phi_{0}
$$

By integrating (6) by $z$ under the conditions (7), the following equation is obtained:

$$
\frac{\rho g z^{2}}{2 \gamma}=1+\operatorname{Sign}(z) \frac{f^{\prime}(z)}{\sqrt{1+f^{\prime}(z)^{2}}}=1-\cos \theta
$$


where $\theta$ is the slope of the water surface $\left(f^{\prime}(z)=\cot \theta\right)$. Then, the following equations can be derived from (8).

$$
\begin{gathered}
z=-\operatorname{Sign}(\theta) L_{c} \sqrt{2(1-\cos \theta)} \\
L_{c}=\sqrt{\frac{\gamma}{\rho g}} \\
f^{\prime}(z)=\frac{2 L_{c}{ }^{2}-z^{2}}{-z \sqrt{4 L_{c}^{2}-z^{2}}}
\end{gathered}
$$

where $L_{c}$ is the capillary length. By integrating (11) by $z$, the equation of the surface profile of the water is given analytically:

$$
x=f(z)=L_{c} \cosh ^{-1}\left(\frac{2 L_{c}}{|z|}\right)-\sqrt{4 L_{c}^{2}-z^{2}}+C
$$

The integration constant $C$ can be determined from the boundary conditions (7). Figure 3 shows the water surface profile given by (12). Since the maximum one-sided width of a water dimple or bump is approximately $10 \mathrm{~mm}$, the maximum lift force of two supporting legs whose spacing is less than $20 \mathrm{~mm}$ decreases due to two water dimples overlapping with one another.

From (3), the force due to surface tension $F_{S}$ reaches a maximum value $2 \gamma$ at $\theta_{0}=\pi / 2$ if the surface of the supporting leg is hydrohphobic $\left(\theta_{c}>\pi / 2\right)$. Under this condition, the depth of the three-phase contact line is $\sqrt{2} L_{c}$, as shown in Fig.4 (a).

$$
\begin{array}{r}
\left(F_{S}\right)_{\max }=2 \gamma=0.146 \mathrm{~N} / \mathrm{m} \quad\left(\text { at } 20^{\circ} \mathrm{C}\right) \\
z_{0}=-\sqrt{2} L_{c}=-3.86 \mathrm{~mm} \quad\left(\text { at } 20^{\circ} \mathrm{C}\right)
\end{array}
$$

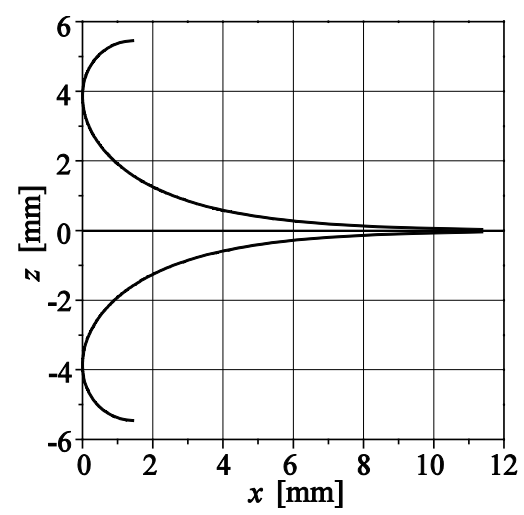

Fig. 3. Profile of the dimple and the bump of water. 


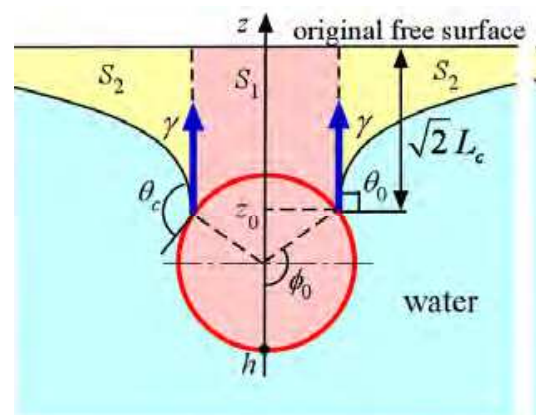

(a) Maximum surface tension force of a hydrophobic leg

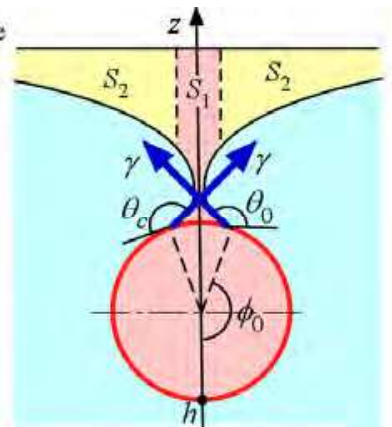

(b) Maximum depth of a hydrophobic leg

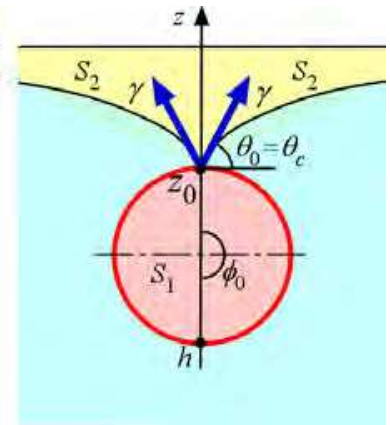

(c) Maximum depth of a hydrophilic leg

Fig. 4. Water breaking conditions

Both the maximum surface tension force and the depth of the leg do not depend on the diameter of the leg or the contact angle. In contrast, the buoyancy force $F_{B}$ does depend on the diameter of the supporting leg. When the diameter is much smaller than $L_{c}$, the force due to surface tension dominates over the buoyancy force. As the depth of the three-phase contact line exceeds $\sqrt{2} L_{c}, \theta_{0}$ becomes greater than $\pi / 2$, and the surface tension force decreases with increasing depth. Figure 4 (b) shows the overhanging water surface just before the surface is broken.

When the surface of the supporting leg is hydrophlic $\left(\theta_{0}<\pi / 2\right)$, the maximum surface tension force is $2 \gamma \sin \theta_{c}$, which decreases with decreasing the contact angle $\theta_{c}$ (Fig. 4 (c)).

\subsection{Pull-off force}

When the leg is lifted out of the water, water rises with the leg, as shown in Fig. 5 (a). Both the buoyancy force and the force due to surface tension, given by (2) and (3), respectively, become negative, that is, downward forces. In this paper, the force needed to lift the leg from the water is defined as the pull-off force. Figure 5 (b) shows the water surface profile just before the leg is completely pulled off when the surface of the supporting leg is hydrophobic. In this situation, the buoyancy force becomes zero, and the maximum pull-off force is given by (15)

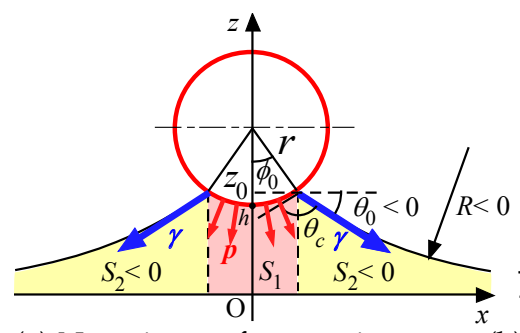

(a) Negative surface tension and buoyancy forces

Fig. 5. Pull-off force

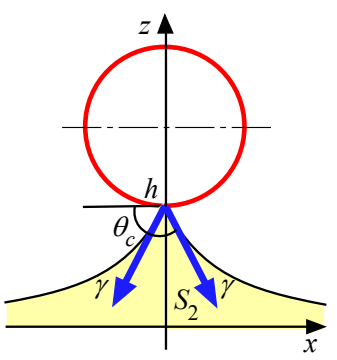

(b) Maximum pull-off force of a hydropobic leg

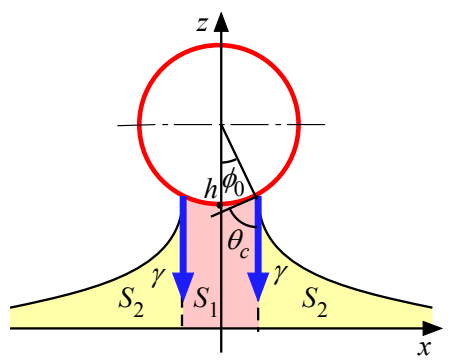

(c) Maximum pull-off force of a hydrophilic leg 


$$
F=2 \gamma \cos \theta_{c}<0
$$

Equation (15) indicates that a leg with a large contact angle can easily be lifted from the water surface. Therefore, super-hydrophobic legs of a water strider reduce the pull-off force instead of generating a large lift force. When the surface of the supporting leg is hydrophilic, the maximum pull-off force is approximately $2 \gamma$ if the surface tension effect is dominant, which does not depend on the contact angle $\theta_{c}$, as shown in Fig. 5 (c).

\subsection{Results of the simulations}

Using the theoretical model shown in the previous section, the relationship between the height of the supporting leg, $h$, and the force acting on the leg, $F$, was investigated, where $h$ is defined as the height of the bottom of the supporting leg from the free water surface, given by:

$$
\begin{aligned}
h & =z_{0}-r\left(1-\cos \phi_{0}\right) \\
& =-\operatorname{Sign}\left(\theta_{0}\right) L_{c} \sqrt{2\left(1-\cos \theta_{0}\right)}-r\left(1-\cos \phi_{0}\right)
\end{aligned}
$$

The force $F$ can be obtained by equations (1) through (4). Thus, $h$ and $F$ are connected by the parameter $\theta_{0}$.

The results of the calculations are shown in Fig.6. Figure 6 (a) demonstrates the effects of the contact angle of the leg surface on the lift and pull-off forces. The pull-off force is shown as the negative lift force. The results show that the lift force of the hydrophobic leg $\left(\theta_{c}>90^{\circ}\right)$ does not differ much due to the contact angle of the leg. In contrast, both the pull-off force and the height where the leg is completely pulled off increase as the contact angle decreases. In the case of th hydrophilic leg $\left(\theta_{c}<90^{\circ}\right)$, the lift force decreases as the contact angle decreases and the pull-off force is almost constant. Figure 6 (b) shows the effects of the diameter of the hydrophobic supporting leg on the lift and pull-off forces. The component of buoyancy force $\left(F_{B}\right)$ is also shown in the same figure. The lift force increases as the diameter of the leg increases. The differences in lift force are derived from the differing buoyancy forces $\left(F_{B}\right)$ that depend on the leg diameter. When the diameter of the leg is less than $0.5 \mathrm{~mm}$, the buoyancy force becomes negligibly small. The force due to surface tension, $F_{S}$, does not depend on the diameter and the contact angle of the leg, because the maximum surface tension force is $2 \gamma$ per unit length if the leg surface is hydrophobic. The buoyancy force, however, is almost canceled by the weight of the leg itself if the specific gravity of the leg is greater than 1. Consequently, the increase in buoyancy force does not necessarily lead to an increase in the net loading capacity. In the case of water strider, the water-repellent hairy legs efficiently increase the loading capacity because water cannot enter the spacing between hairs, and so the apparent diameter is enlarged without increasing the mass of the leg. 


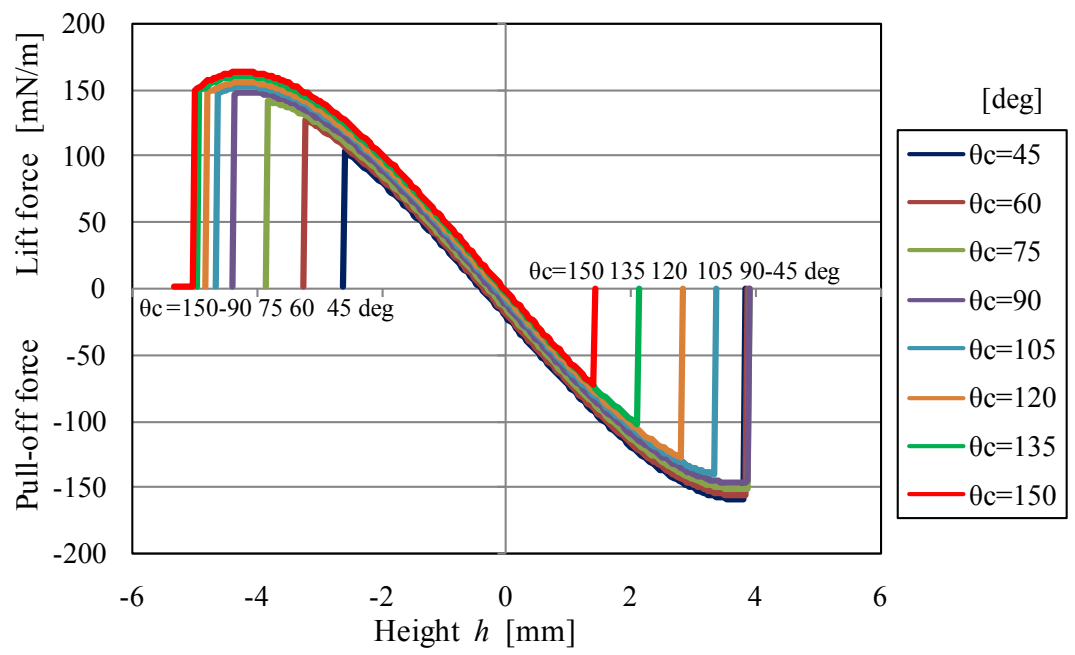

(a) Effect of contact angle on the lift and pull-off forces

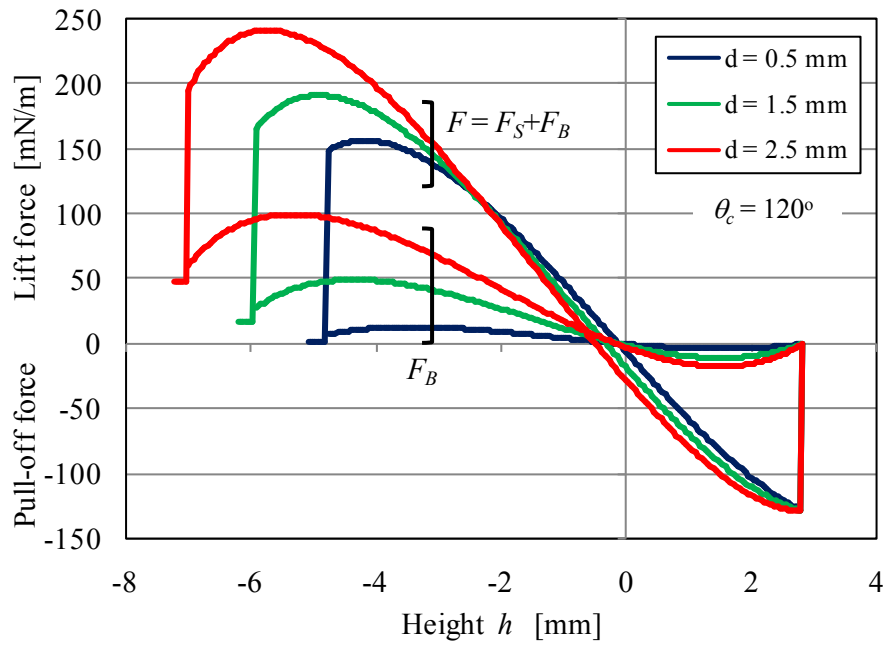

(b) Effect of diameter of supporting leg on the lift and pull-off forces

Fig. 6. Results of the simulations 


\section{Fabrication Of The Microstructured Legs}

According to Wenzel's law and Cassie-Baxter's law, micro structures on a surface enhance hydrophobicity. Mechanical structures as well as chemical properties help create superhydrophobic surfaces. In the present paper, three kinds of hydrophobic supporting legs with micro structures fabricated using MEMS processes are proposed.

Figure 7 shows a PDMS (polydimethlysiloxane) hair-like structure wrapped on a $0.5-\mathrm{mm}$ diameter brass wire. The process starts with the fabrication of a mold by the patterning of SU-8, a photoresist that enables the creation of thick patterns by UV lithography (Fig.7 (a)). Then PDMS is poured into the grooves of the mold by capillary action, cured, and released from the mold to form the comb-shaped structure shown in Fig.7 (b). The PDMS structure is wrapped around the wire and adhered by a two-component epoxy adhesive. As the last step, the structure is dipped into a fluorinated hydrophobic agent (Fluoro Technology, FS1010) to coat the surface of the structure.

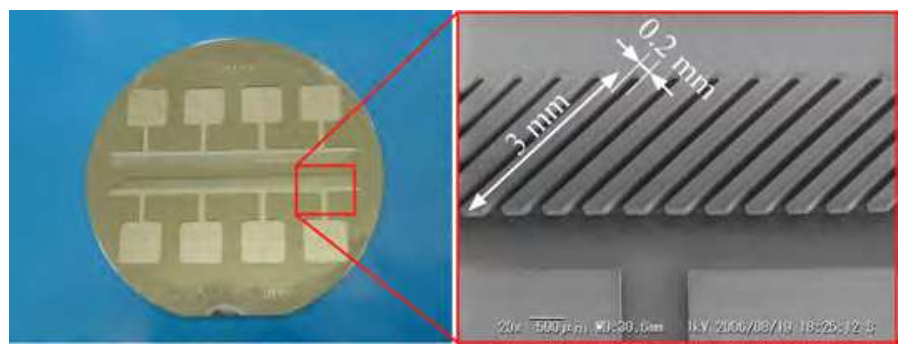

(a) SU8 mold for PDMS structure

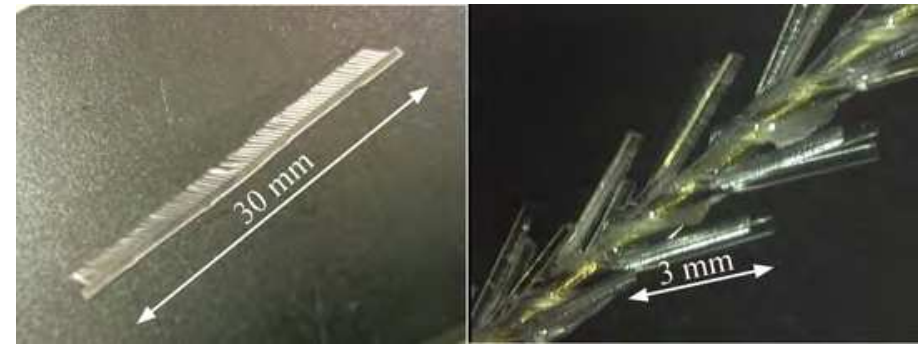

(b)PDMS comb-shaped structure (c) PDMS structure wrapped on a wire

Fig. 7. PDMS hair-like structure

The second structure, shown in Fig. 8, consists of SU-8 patterns fabricated by photolithography on a 1-mm-diameter cylindrical brass wire. The process of exposure is shown in Fig.8 (a). An $80-\mu$ m-thick SU-8 layer is coated on the wire by dipping and the surface of the SU-8 is divided into 5 faces, with each face exposed separately. Circular patterns with a diameter of $100 \mu \mathrm{m}$ are formed by developing the SU8 layer (Fig.8 (b)). Then, the same hydrophobic agent (FS-1010) is coated on the structure by dipping. SEM micrographs of the SU-8 structure are shown in Fig.8 (c). 


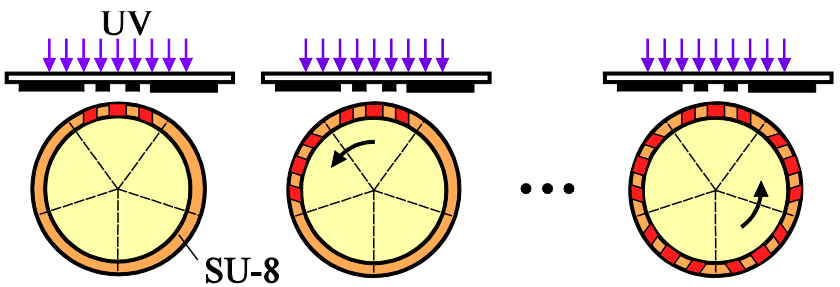

(a) Photolithography on the cylindrical surface

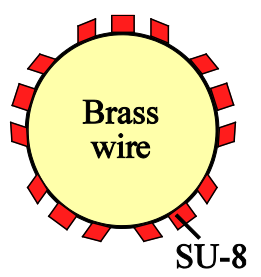

(b) Development

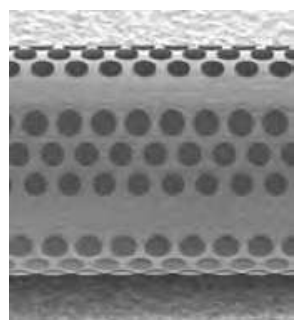

(c) SEM images of SU-8 structure

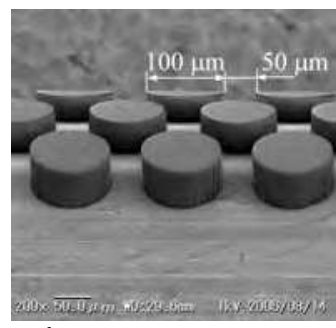
(1)

Fig. 8. SU-8 structure

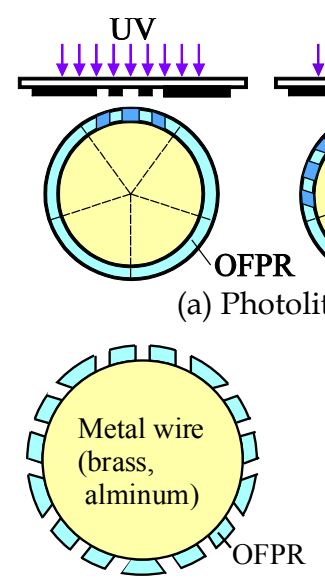

(b) Development
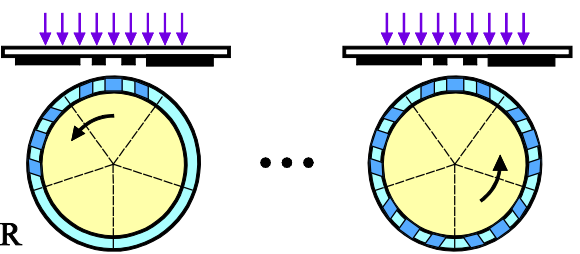

(a) Photolithography on a metal wire

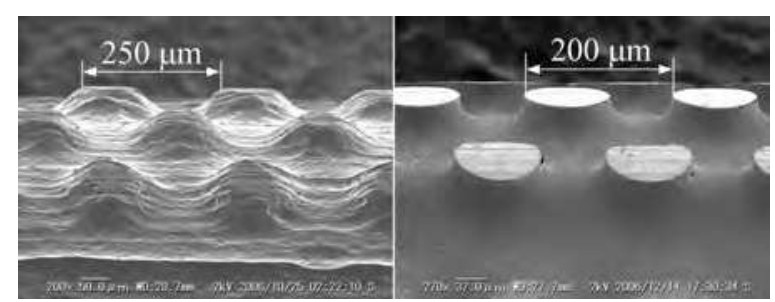

$\begin{array}{ll}\text { (e) Aluminum structure } & \text { (f) Brass structure }\end{array}$

Fig. 9. Wet etching of aluminum and brass wires 
The third structure is formed on the surfaces of aluminium and brass wires by wet etching. Figure 9 shows the process to fabricate the etched structures. First, photolithography on the cylindrical metal wires was carried out in the same manner as in the SU8 structure. Here, a positive type photoresist (Tokyo Ohka Kogyo, OFPR) was used instead of SU-8 (Fig. 9 (a) (b)). The wires were then patterned by isotropic wet etching (Fig. 9 (c)), followed by removal of the OFPR (Fig. 9 (d)). Finally, the same hydrophobic agent (FS-1010) was coated on the structure. SEM photographs of the aluminium and brass structures are shown in Fig. 9 (e) and (f), respectively. The brass structures have sharper edges than do the aluminium structures.

\section{MEASUREMENTS OF LIFT AND PULL-OFF FORCES}

\subsection{Experimental setup}

To investigate the performance of the supporting legs, the lift and pull-off forces of the fabricated legs were measured. The experimental setup for the measurements is illustrated in Fig. 10. The geometry of the specimen is shown in Fig.11. Both ends of the specimen are bent up in order to prevent the tip of the wire from breaking the water surface.

The surface of the water in a petri dish was moved vertically using a $z$ stage to immerse and pull out the specimen. The lift and pull-off forces were measured by using a laser displacement sensor to detect the deformation of a parallel leaf spring fixed to the specimen. By using two laser displacement sensors, the relative height of the specimen from the water surface was also measured. Table I shows materials, surface structures, outer diameters, and contact angles of the specimens. Three kinds of the hydrophobic-agent-coated specimens, as well as four specimens with microfabricted structures on their surfaces, were prepared. The contact angles of the microfabricated wires, except for the PDMS structure, increased by 5-10 degrees compared to those of the FS-1010 coated non-structured wire. The PDMS structure was too large to show an increase in the contact angle.

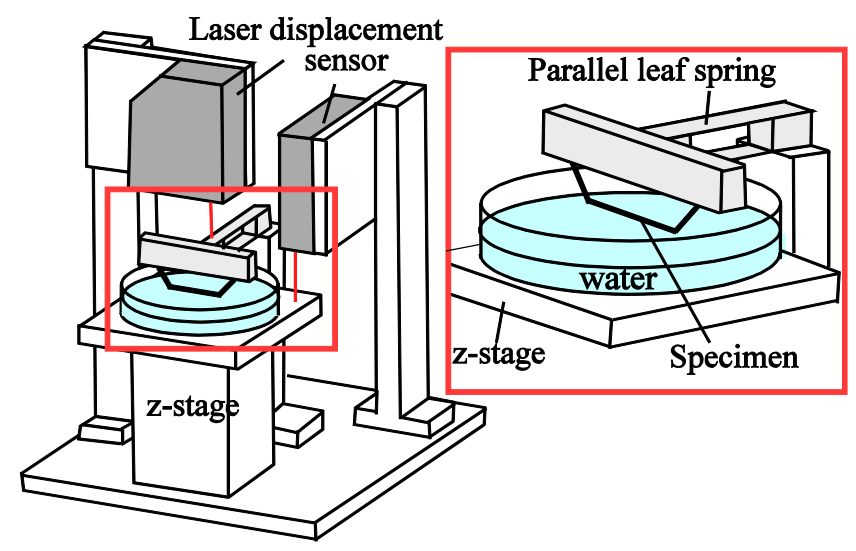

Fig. 10. Experimental setup for measuring lift and pull-off forces 


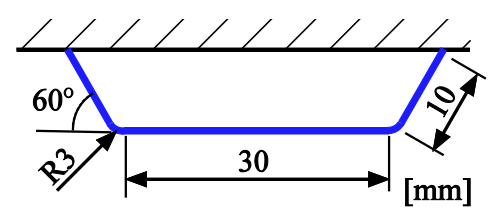

Fig. 11. Geometry of the specimen

\begin{tabular}{|c|c|c|c|c|}
\hline Base wire & $\begin{array}{c}\text { MEMS } \\
\text { structure }\end{array}$ & $\begin{array}{c}\text { Hydrophobic } \\
\text { coating }\end{array}$ & $\begin{array}{c}\text { Outer } \\
\text { Diameter }\end{array}$ & $\begin{array}{c}\text { Contact } \\
\text { angle }\end{array}$ \\
\hline Brass $\phi 0.5$ & -- & FS-6130*1 & $0.5 \mathrm{~mm}$ & $105^{\circ}$ \\
\hline Brass $\phi 0.5$ & -- & FS-1010 ${ }^{\star} 1$ & $0.5 \mathrm{~mm}$ & $118^{\circ}$ \\
\hline Brass $\phi 0.5$ & -- & HIREC-1450*2 & $0.62 \mathrm{~mm}$ & $135^{\circ}$ \\
\hline Brass $\phi 0.5$ & PDMS & FS-1010*1 & $2.5 \mathrm{~mm}$ & $117^{\circ}$ \\
\hline Brass $\phi 1.0$ & SU-8 & FS-1010*1 & $1.1 \mathrm{~mm}$ & $128^{\circ}$ \\
\hline $\begin{array}{c}\text { Aluminium } \\
\phi 1.4 \\
\end{array}$ & Etching & FS-1010*1 & $1.4 \mathrm{~mm}$ & $123^{\circ}$ \\
\hline Brass $\phi 1.0$ & Etching & FS-1010*1 & $1.0 \mathrm{~mm}$ & $123^{\circ}$ \\
\hline
\end{tabular}

*1 Fluoro Technology Corp. *2 NTT Advanced Technology Corp.

Table 1. Material, Diameter, and Contact Angle of the Specimens

\subsection{Experimental results}

Figure 12 shows the relation of height $h$ with lift and pull-off forces for specimens without MEMS structures. The results for the specimens with MEMS structures are shown in Fig.13. Experimental and calculated data are shown in (a) and (b), respectively, in both figures. In the experiment, the supporting leg was first immersed gradually into water. In this process, the lift force initially increased, reached a maximum, and then decreased slightly; finally, the water's surface was broken and the leg was completely submerged. After that, as the leg was pulled out of the water, the leg remained submerged until it came close to the surface of water. Then, a dimple of water formed abruptly, and it was gradually raised along with the specimen. This hysteresis is observed in the experimental results shown in Figs 12 (a) and 13 (a), although this effect was not taken into account in the calculations.

The measured lift forces are in good agreement with the calculated values. The maximum lift force depends on the diameter of the specimens rather than the surface properties of the legs. The measured pull-off force decreases with increasing contact angle. This trend agrees well with the theoretical predictions. However, the measurements for the values of the maximum pull-off force and maximum height are smaller than those calculated. This is because the meniscus bridge shrinks at one end of the leg and becomes a conical shape just before the leg is completely pulled off. Therefore, a three dimensional dynamic model of the meniscus is necessary to predict the maximum pull-off force quantitatively.

The PDMS hair-like structure wrapped on the wire of $0.5 \mathrm{~mm}$ in diameter enlarges to an apparent diameter of $2.5 \mathrm{~mm}$ and increases the maximum possible lift force efficiently, even though it does not improve the contact angle and pull-off force. 


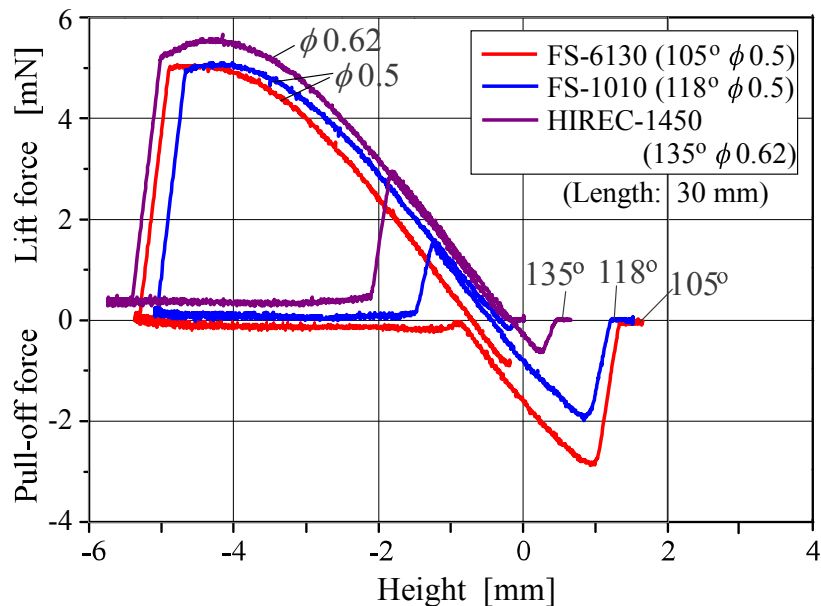

(a) Experimental results

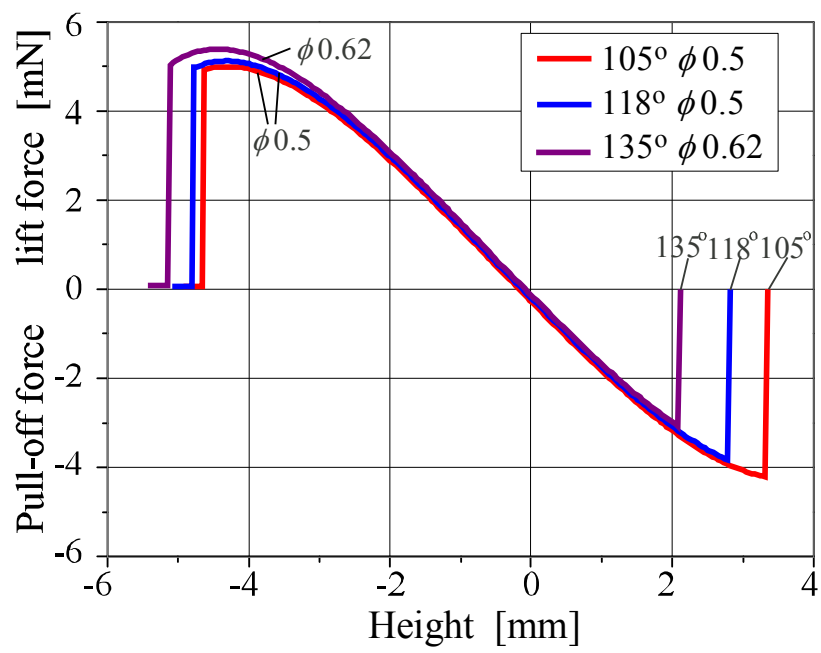

(b) Calculated results

Fig. 12. Lift and pull-off forces for hydrophobic-agent-coated legs 


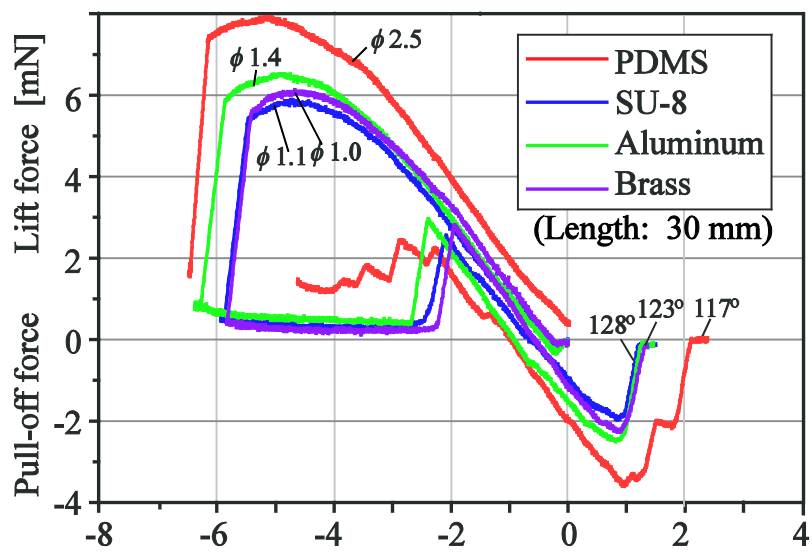

Height [mm]

(a) Experimental results

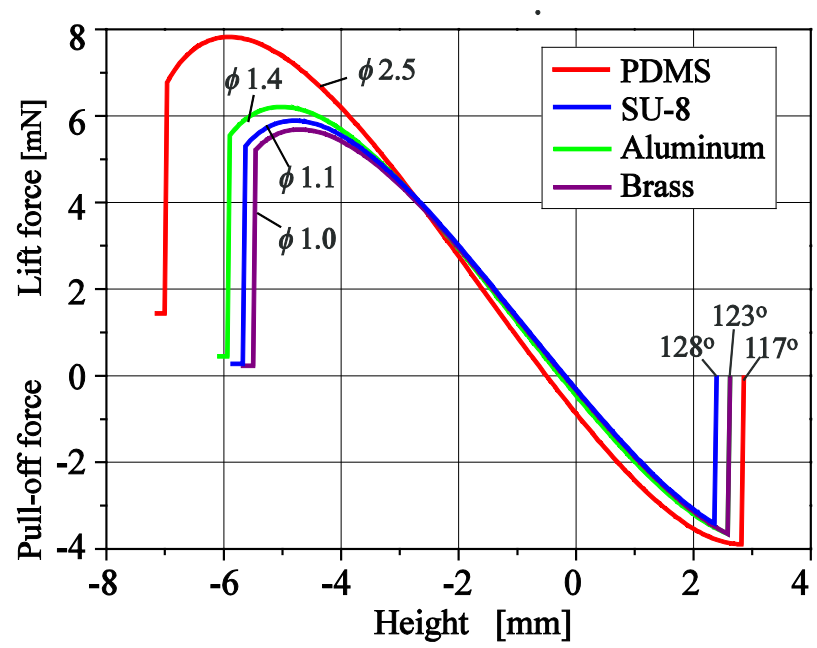

(b) Calculated results

Fig. 13. Lift and pull-off forces for legs with microfabricated structures

\section{DEVELOPMENT OF WATER STRIDER ROBOTS}

\subsection{Hexapedal robot}

Two different mechanisms for surface-tension-based locomotion on the water surface were developed. Figure 14 shows a hexapedal robot. The hydrophobic legs with PDMS hair-like structures were used for the forelegs and hind legs to support the weight of the body. The middle legs were attached to an actuating mechanism that creates the elliptical motion required for propulsion. The resulting hexapedal locomotion is similar to that of an insect water strider. Each supporting leg is $135 \mathrm{~mm}$ in length. From the results of Fig. 13 (a), the loading capacity of the four supporting legs was predicted to be $138 \mathrm{mN}$ (14 gf), which is 
sufficient to support a robot that weighs 5.4 gf. A DC motor and a lithium polymer battery were mounted on the body, and non-tethered actuation was achieved.

The slider-crank mechanism, shown in Fig. 15, was used for creating the elliptical motion of the middle legs. The trajectory of point $\mathrm{P}$ at the middle of the connecting rod is given by the following equations:

$$
\left\{\begin{array}{l}
x=r \cos \theta+\left(1-\frac{a}{l}\right) \sqrt{l^{2}-r^{2} \sin ^{2} \theta} \\
y=\frac{a r}{l} \sin \theta
\end{array}\right.
$$

Definitions of $r, l, a, \theta \square$ and the calculated trajectory of $\mathrm{P}$ is shown in Fig. 16. Figure 17 illustrates the transmission mechanism for the middle leg. The 125-mm-long middle leg penetrates the connecting rod at $\mathrm{P}$ and is supported at $\mathrm{Q}$ using a flexible ring, so that the middle leg rotates conically around $Q$. The rowing angle of the leg is approximately $90^{\circ}$ and the elliptical motion of $\mathrm{P}$ is magnified by 10 at the tip of the leg.

The hexapedal robot was put on the surface of water in a container and the middle legs were driven at $2 \mathrm{~Hz}$. Forward motion was successfully achieved on the surface down to 5-mmdeep water. The velocity determined from the images of a high-speed camera is shown in Fig. 18 (a). A maximum speed of $90 \mathrm{~mm} / \mathrm{s}$ and an average speed of $40 \mathrm{~mm} / \mathrm{s}$ were achieved. The middle legs were swept shallowly without breaking the surface of water and pulled off during the power stroke. Consequently, deceleration starts in the middle of the power stroke. Then, acceleration starts in the middle of the recovery stroke because of the inertial force of the actuating legs. The velocity of an actual water strider obtained from high-speed video images is shown in Fig.18 (b). The water strider accelerates all the way through the power stroke and reaches a maximum speed of about $400 \mathrm{~mm} / \mathrm{s}$. Then it decelerates rapidly in the recovery stroke. After that, it glides on the surface of the water, decelerating moderately.

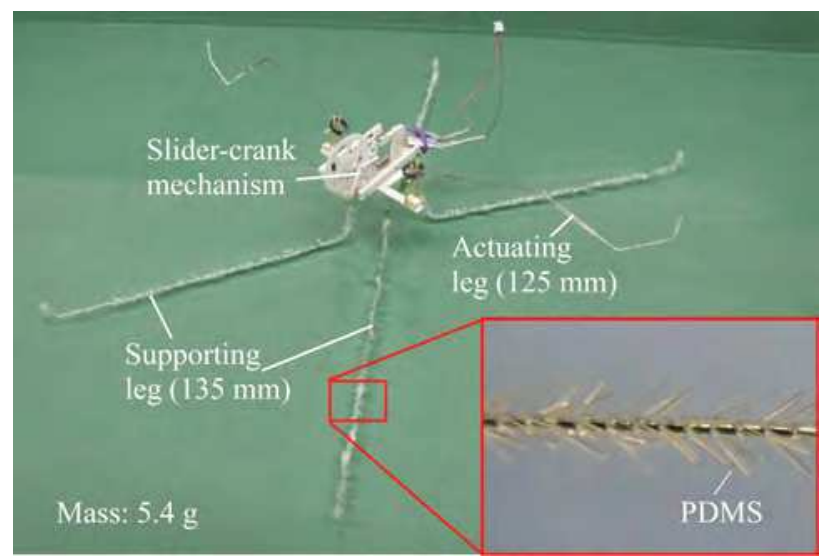

Fig. 14. Hexapedal water strider robot 


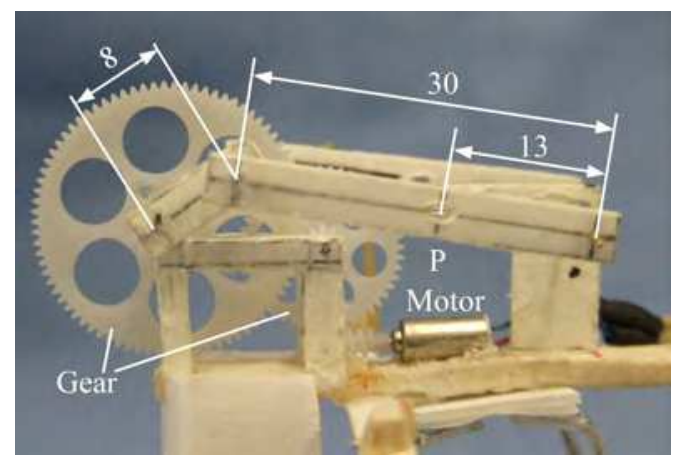

Fig. 15. Slider-crank mechanism for creating elliptical motion of middle leg.

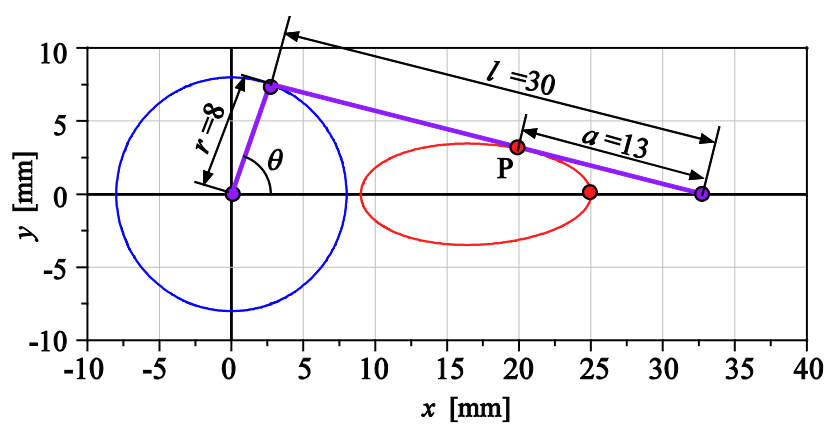

Fig. 16. Elliptical trajectory created by the slider-crank mechanism

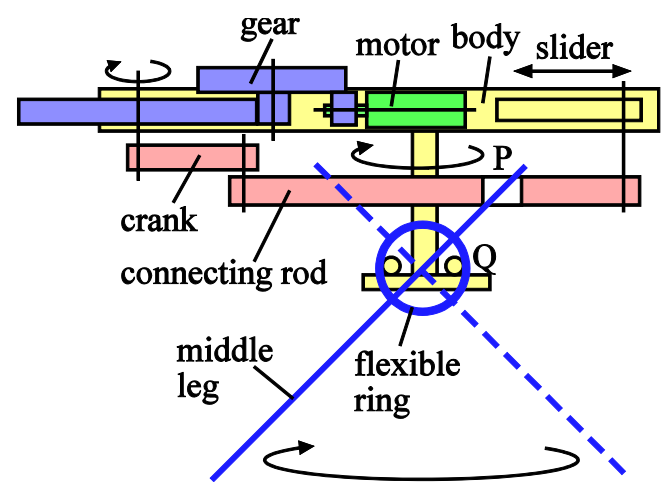

Fig. 17. Structure of the middle leg 


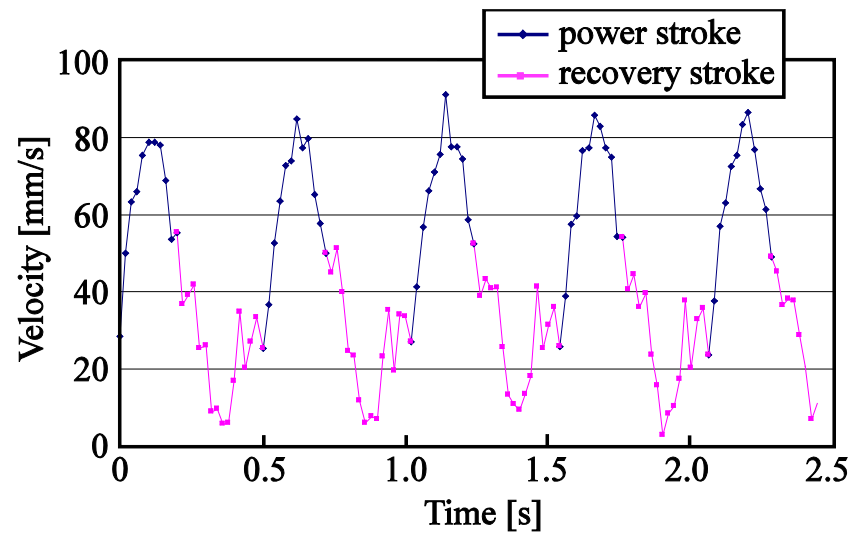

(a) Hexapedal robot

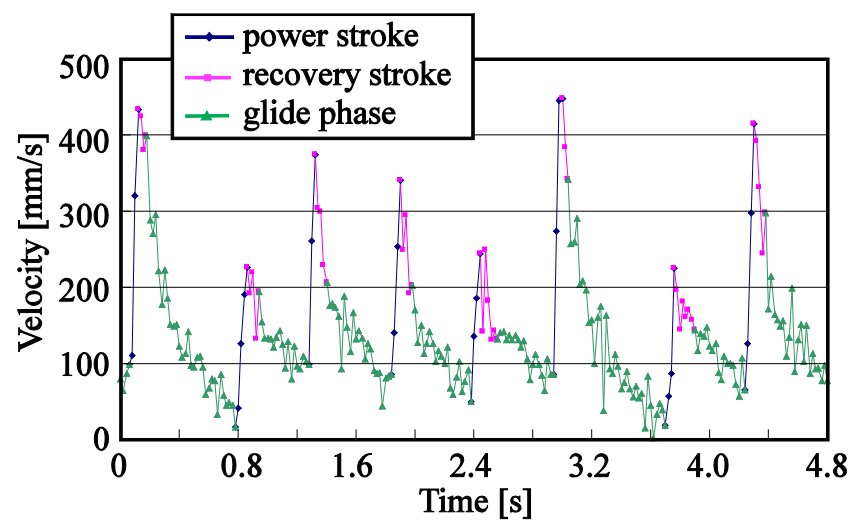

(b) Insect water strider

Fig. 18. Variation of the velocity during the rowing motion

\subsection{Robot with a vibration motor}

The other mechanism developed in the present study was a robot that moves on the water surface by the vibration of twelve supporting legs. A photograph of the robot is shown in Fig. 19. A vibration motor with an eccentric weight is mounted on the body. This type of motor is used in cell phones. Brass legs with wet-etched microstructures are used as supporting legs so that the microstructures can withstand high-frequency vibrations, as shown in the inset of Fig. 19. The geometry of both ends of the leg is the same as those of the specimen shown in Fig. 11. One end of the leg is attached to the robot's body directly. Each leg has a different length and consequently a different resonant frequency. When the rotational speed of the vibration motor is varied, different legs resonate, enabling the robot to move forward, left, and right. Thus, multiple motions can be realized using one motor without any mechanism for power transmission. The actuation method for micro mobile robots utilizing resonance with externally applied vibration was proposed by Yasuda et al. 
(Yasuda et al., 1994). However, a self-contained robot with a vibrator and a power source has not been achieved.

The layout of the legs is shown in Fig.20. The roles and specifications of the legs are shown in Table 2. From the results of Fig.13 (a), total length of twelve legs is $1 \mathrm{~m}$ and the maximum payload is predicted to be $200 \mathrm{mN}$ (20.4gf). A vibration motor, lithium polymer battery, and electronics for PWM (pulse width modulation) control of rotational speed were mounted on the robot for fully autonomous actuation. The robot weighed $7.85 \mathrm{~g}$ and could therefore be supported by the legs with a safety factor of greater than 2 .

Figure 21 shows the measured and calculated resonant frequencies of the 1st and 2nd modes in air and on the surface of the water as a function of leg length. The 2nd-mode vibration was used for locomotion, since the frequency range matches that of the PWM-controlled vibration motor. Calculated resonant frequencies were obtained from the following equation for resonant frequency of a straight cantilever:

$$
f=\left(\frac{\lambda}{L}\right)^{2} \sqrt{\frac{E I}{\rho A}}
$$

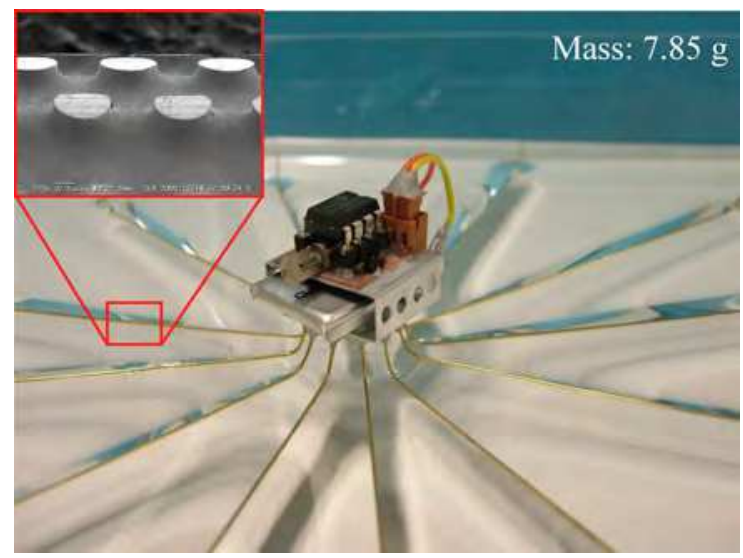

Fig. 19. Water strider robot with a vibration motor

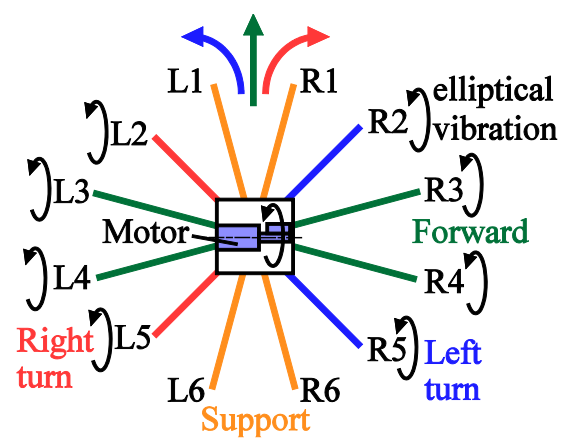

Fig. 20. Layout of the supporting legs. 
where $E$ is Young's modulus, $I$ is the geometrical moment of inertia, $\rho$ is the density, $A$ is the cross sectional area, $L$ is the length of the supporting leg including both angled ends, and $\lambda$ is a constant (1st mode: 1.875, 2nd mode: 4.694). The resonant frequencies in air are in good agreement with the calculated values. The resonant frequencies on the surface of the water are lower than those in air because of the additional mass of water around the leg. Figure 22 shows the trajectory of a 90-mm-long leg (R3) resonated at $120 \mathrm{~Hz}$ in air. The leg rotated elliptically in sync with the eccentric mass of the vibration motor. This elliptical vibration generates the propulsive force on the surface of water

Photographs of the robot moving on the surface of an outdoor pond are shown in Fig. 23. The robot successfully moved forward and rotated left and right in accordance with the embedded program without breaking the surface of the water. The velocity of the forward motion was approximately $70 \mathrm{~mm} / \mathrm{s}$, whereas both the velocity of the left and right turns was $50 \mathrm{~mm} / \mathrm{s}$. The radius of the left and right turns was $700 \mathrm{~mm}$ and $800 \mathrm{~mm}$, respectively. These differences are due to the differences in the number and length of the resonated legs.

\begin{tabular}{|c|c|c|c|c|}
\hline Leg No. & Role & Length & $\begin{array}{c}\text { Resonant } \\
\text { Frequency }\end{array}$ & $\begin{array}{c}\text { Duty factor } \\
\text { of input pulse }\end{array}$ \\
\hline $\begin{array}{c}\text { L-1,6 } \\
\text { R-1,6 }\end{array}$ & Support & $85 \mathrm{~mm}$ & --- & --- \\
\hline $\begin{array}{c}\text { L-3,4 } \\
\text { R-3,4 }\end{array}$ & $\begin{array}{c}\text { Forward } \\
\text { motion }\end{array}$ & $90 \mathrm{~mm}$ & $109 \mathrm{~Hz}$ & $32 \%$ \\
\hline R-2,5 & Left turn & $80 \mathrm{~mm}$ & $115 \mathrm{~Hz}$ & $34 \%$ \\
\hline L-2,5 & Right turn & $70 \mathrm{~mm}$ & $132 \mathrm{~Hz}$ & $39 \%$ \\
\hline
\end{tabular}

Table 2. Role, Length, and Resonant Frequency of the Leg

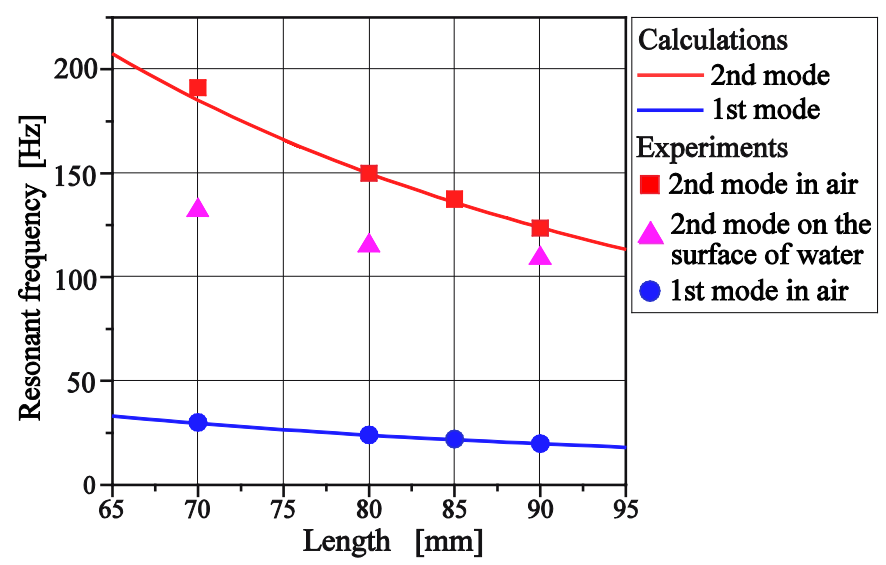

Fig. 21. Resonant frequencies in air and on the surface of water as a function of length of the supporting leg. 


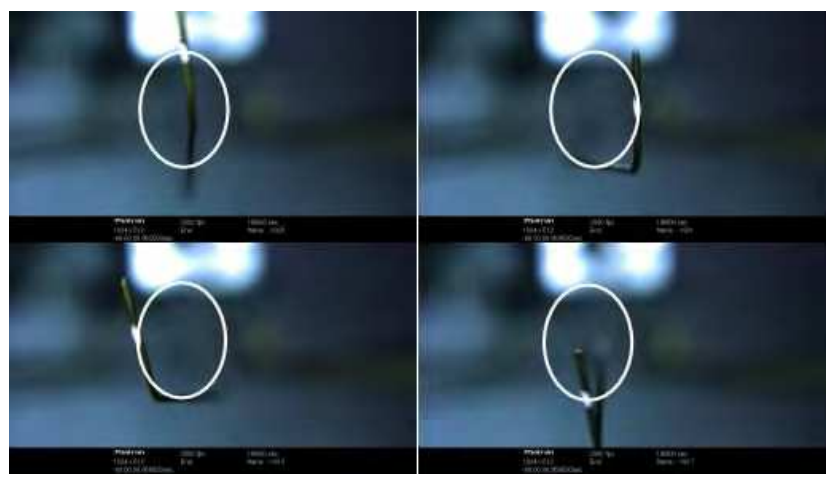

Fig. 22. Trajectory of the supporting leg resonated in air. (Leg No. R3, 120Hz)

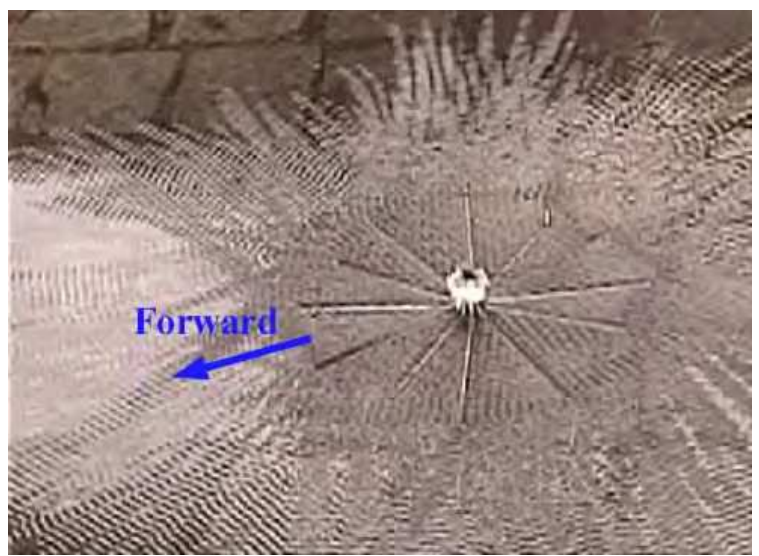

(a) Forward mortion $(109 \mathrm{~Hz})$

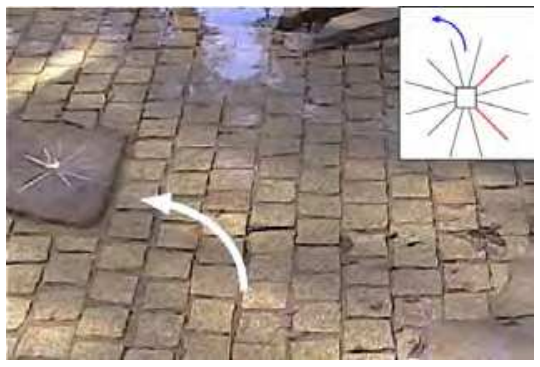

(b) Left turn $(115 \mathrm{~Hz})$

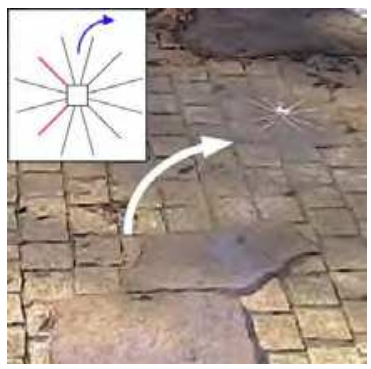

(c) Right turn $(132 \mathrm{~Hz})$

Fig. 23. Locomotion utilizing resonant vibration 


\section{Conclusion}

Motivated by highly optimized working principles of small insects, we have been studying the surface-tension-based locomotion on the surface of water. Various kinds of supporting legs with microfabricated hydrophobic surfaces were developed using MEMS techniques, and the force balance between the stationary legs and the surface of water was clarified both theoretically and experimentally. Two kinds of non-tethered water strider robots with microfabricated supporting legs were developed, and autonomous locomotion on the surface of water was demonstrated. Actuation utilizing resonant vibration was effective in simplifying the mechanisms for miniature mobile robots. Dynamic analysis of the locomotion on the surface of water is left for future research.

Locomotion that depends on surface tension force provides miniature robots with efficient mobility on shallow water. In the near future, such robots with microsensors and wireless communication devices can potentially be used for entertainment and environmental monitoring, such as water quality surveys.

\section{References}

Floyd, S.; Sitti, M. (2008). Design and Development of the Lifting and Propulsion Mechanism for a Biologically Inspired Water Runner Robot, IEEE Transactions on Robotics, Vol. 24, No. 3, pp.698-709.

Floyd, S.; Keegan, T. \& Sitti, M. (2006). A Novel Water Running Robot Inspired by Basilisk Lizards, Proceedings of the IEEE/RSJ Intelligent Robot Systems Conference, Beijing, pp. $5430-5436$.

Gao, X. \& Jiang, L. (2004) Water-repellent legs of water strider, Nature, Vol. 432, p.36.

Glasheen, J. W. \& McMahon, T. A. (1996). A hydrodynamic model of locomotion in the Basilisk Lizard, Nature, Vol. 380, pp.340-342.

Glasheen, J. W. \& McMahon, T. A. (1996). Size-Dependence of Water- Running Ability in Basilisk Lizards, Journal of Experimental Biology, vol. 199, pp. 2611-2618.

Hu, D. L.; Xhan, B. \& Bush, J. W. M. (2003) The hydrodynamics of water strider locomotion," Nature, Vol. 424, pp.663-666, 7.

Keller, J. B. (1998). Surface Tension Force on a Partially Submerged Body, Physics of Fluids, Vol. 10, pp. 3009-3010.

Song, Y. S. ; Suhr, H. \& Sitti, M. (2006). Modeling of the Supporting Legs for Designing Biomimetic Water Strider Rob.ots, Proceedings of IEEE Int. Conf. on Robotics and Automation, Orlando, pp.2303-2310.

Song, Y. S. \& Sitti, M. (2007)a. Surface-Tension-Driven Biologically Inspired Water Strider Robots: Theory and Experiments, IEEE Transactions of Robotics, Vol. 23, No. 3 pp.578-589.

Song, Y. S. \& Sitti, M. (2007)b. STRIDE: A Highly Maneuverable and Non- Tethered Water Strider Robot, Proceedings of IEEE Int. Conf. Robotics and Automation, Roma, 2007, pp.980-984.

Suhr, S. H.; Song, Y., S; Lee, S. J. \& Sitti, M. (2005) Biologically Inspired Water Strider Robot, Robotics: Science and Systems, Boston, pp. 319-325. 
Suter, R. B. \& Wildman, H. (1997). Locomotion on the Water Surface: Propulsive Mechanisms of the Fisher Spider DOLOMES TRITON, Journal of Experimental Biology, Vol. 200, pp. 2523-2538.

Suter, R. B.; Rosenberg, O.; Loeb, S.; Wildman, H. \& Long, J. H. Jr. (1999). “Locomotion on the Water Surface: Hydrodynamic Constraints on Rowing Velocity Require a Gait Change, Journal of Experimental Biology, Vol. 202, pp. 2771-2785.

Suzuki, K.; Shimoyama, I. \& Miura, H. (1994). Insect-Model Based Microrobot with Elastic Hinges, IEEE J. Microelectromecanical Systems, Vol. 3, No. 1, pp. 4-9, March 1994.

Suzuki, K.; Takanobu, H.; Noya, K.; Koike, H. \& Miura, H. (2007). Water Strider Robots with Microfabricated Hydrophobic Legs, Proceedings of. IEEE/RSJ Int. Conf. Intelligent Robots and Systems (IROS07), San Diego, pp.590-595.

Wood, R. J (2008). The First Takeoff of a Biologically Inspired At-Scale Robotic Insect, IEEE Transactions of Robotics, Vol. 24, No. 2, pp.341-347.

Yasuda, T.; Miura, H. \& Shimoyama, I. (1994). Microrobot Actuated by a Vibration Energy Field, Sensors and Actuators, A: physical, Vol. 43, No. 1-3, pp.366-370. 


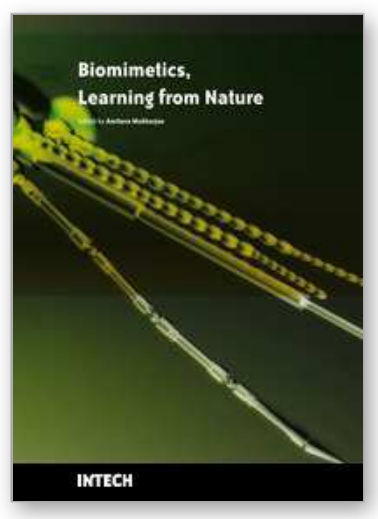

\section{Biomimetics Learning from Nature}

Edited by Amitava Mukherjee

ISBN 978-953-307-025-4

Hard cover, 534 pages

Publisher InTech

Published online 01, March, 2010

Published in print edition March, 2010

Nature's evolution has led to the introduction of highly efficient biological mechanisms. Imitating these mechanisms offers an enormous potential for the improvement of our day to day life. Ideally, by bio-inspiration we can get a better view of nature's capability while studying its models and adapting it for our benefit. This book takes us into the interesting world of biomimetics and describes various arenas where the technology is applied. The 25 chapters covered in this book disclose recent advances and new ideas in promoting the mechanism and applications of biomimetics.

\section{How to reference}

In order to correctly reference this scholarly work, feel free to copy and paste the following:

Kenji Suzuki (2010). Bio-Inspired Water Strider Robots with Microfabricated Functional Surfaces, Biomimetics Learning from Nature, Amitava Mukherjee (Ed.), ISBN: 978-953-307-025-4, InTech, Available from: http://www.intechopen.com/books/biomimetics-learning-from-nature/bio-inspired-water-strider-robots-withmicrofabricated-functional-surfaces

\section{INTECH}

open science | open minds

\section{InTech Europe}

University Campus STeP Ri

Slavka Krautzeka 83/A

51000 Rijeka, Croatia

Phone: +385 (51) 770447

Fax: +385 (51) 686166

www.intechopen.com

\section{InTech China}

Unit 405, Office Block, Hotel Equatorial Shanghai

No.65, Yan An Road (West), Shanghai, 200040, China

中国上海市延安西路65号上海国际贵都大饭店办公楼 405 单元

Phone: +86-21-62489820

Fax: +86-21-62489821 
(C) 2010 The Author(s). Licensee IntechOpen. This chapter is distributed under the terms of the Creative Commons Attribution-NonCommercialShareAlike-3.0 License, which permits use, distribution and reproduction for non-commercial purposes, provided the original is properly cited and derivative works building on this content are distributed under the same license. 